\title{
El uso del polígrafo en los procesos de selección laboral: un caso de vacío legal en el sistema jurídico colombiano ${ }^{1}$
}

The use of polygraph for recruitment: a case of loophole in colombia

Oscar Andrés López Cortés ${ }^{2}$

Fecha de recepción: 2 de mayo de 2015

Fecha de aprobación: 7 de junio 2015

\section{RESUMEN}

Este artículo analiza el uso del polígrafo en los procesos de selección laboral, bajo la condición de que no existe norma en el derecho colombiano que lo permita pero tampoco una que lo prohíba. La pregunta indaga si conforme al principio de dignidad humana y los derechos fundamentales en el trabajo es posible o no emplear el polígrafo en la selección de personal. Para ello, en el presente artículo se reconstruirá el marco legal colombiano sobre el polígrafo, posteriormente, se plantearán tres argumentos de corte axiológico que permiten concluir razonablemente que el uso del polígrafo en la selección de personal no es constitucional.

Palabras clave: polígrafo, selección de personal, dignidad humana.

1 Este artículo hace parte del proyecto Análisis de los convenios fundamentales de la OIT y sus efectos en la cultura jurídica laboral, adelantado por el grupo de investigación Derecho Laboral y Estándares Internacionales de la Universidad Libre, Seccional Bogotá, y se desarrolla como parte de la línea de investigación Derechos Fundamentales en las Formas Contemporáneas de Trabajo.

2 Ph.D. en Antropología de la Universidad del Cauca (Colombia), Magister en Derecho de la Universidad Nacional de Colombia, Especialista en Derecho del Trabajo de la Universidad Nacional de Colombia, Abogado de la Universidad Nacional de Colombia. Docente Investigador de jornada completa de la Universidad Libre. Responsable de la Línea de Investigación Derechos Fundamentales en las formas contemporáneas del trabajo. Correo electrónico institucional: oscara.lopezc@unilibrebog.edu.co 


\begin{abstract}
This article analyzes the use of polygraph for recruitment, subject that there is a loophole about this in Colombia. The question inquires if in accordance to human dignity principle and Fundamental Rights at Work is possible to use the polygraph for recruitment or not. Therefore, the Colombian legal frame about polygraph will be reconstructed; later three axiological arguments that will allow to reasonably conclude that the polygraph use for recruitment is not constitutional will be exposed.
\end{abstract}

Keywords: Polygraph, recruitment, human dignity 


\section{INTRODUCCIÓN}

¿Ha usted consumido alucinógenos?, ¿tiene usted relación familiar con alguna persona al margen de la ley?, ¿alguna vez ha robado? Estas y otras preguntas son realizadas, cada vez con más frecuencia, a quienes aspiran a ser seleccionados como cajeros de bancos, personal de aseo en hospitales, abogados de entidades públicas, guardias de seguridad, asistentes contables, etc. Sin importar la naturaleza de la organización, las funciones a realizar, el perfil requerido, incluso, cualquiera sea la forma de vinculación, muchas personas son colocadas ante el polígrafo como parte del proceso de selección laboral.

El polígrafo ha tenido desde su creación diferentes aplicaciones, pero son dos de ellas las que podrían considerarse usuales: en materia de investigación criminal y en procesos de selección de personal. En Colombia, la oferta de empresas que aseguran tener el polígrafo más avanzado y el personal técnico mejor entrenado para su aplicación va en aumento, no obstante, que se trata de un servicio cuya regulación legal es prácticamente inexistente, al menos, en lo que a asuntos laborales se refiere, pues en materia penal ya se cuenta con un pronunciamiento de la Corte Suprema de Justicia que proscribe su uso.

Sin embargo, que en materia de selección de personal carezcamos de expresa regulación legal para el uso del polígrafo, no significa la inexistencia de argumentos jurídicos para determinar si su uso debería estar permitido en Colombia, aspecto que demarca el objetivo central de este artículo. Así, la pregunta que orienta el análisis es la siguiente ¡es en Colombia constitucionalmente admisible el uso del polígrafo dentro de un proceso de selección de personal, o por el contrario, una lectura del ordenamiento jurídico proscribe su empleo con semejante propósito? Para responderla se procederá así: se describirá el marco legal existente en Colombia respecto al polígrafo en Colombia, en el segundo apartado se desarrollará la discusión de orden constitucional relacionada con la dignidad humana en el trabajo como marco iusfilosófico al cual se debe ajustar el proceso de selección de personal para el trabajo, y finalmente, se presentarán las conclusiones del análisis. 


\section{Desarrollo}

\section{El marco legal}

El uso del polígrafo dentro de un proceso de selección de personal ha sido reglamentado en dos casos: en la Unidad Administrativa Especial Dirección de Impuestos y Aduanas Nacionales (en adelante DIAN) conforme a la Resolución 7635 publicada en el Diario Oficial 46681; y en las empresas vigiladas por la Superintendencia de Vigilancia Privada según lo señalado en las resoluciones 2592 de 2003 -publicada en el Diario Oficial 45412- y 2417 de 2008 -publicada en el Diario Oficial 47045-, por medio de las cuales se autoriza y regula la utilización del polígrafo por parte de los Servicios de Vigilancia y Seguridad Privada en Colombia.

Frente al primer caso es fácil encontrar suficientes razones que justifican su uso, no solo por la delicada labor constitucionalmente asignada a la DIAN, sino por la cantidad de escándalos de corrupción que han rodeado a esa entidad pública ${ }^{3}$. Por supuesto, el uso del polígrafo como herramienta de selección de personal y control periódico de las conductas de los funcionarios que allí laboran, no garantiza la disminución de los índices de corrupción al interior de la DIAN.

El segundo caso no es tan sencillo. El rango de empresas bajo la órbita de control de la Superintendencia de Vigilancia Privada es muy amplio y cubre diferentes tipos de servicios: la seguridad de bienes muebles e inmuebles, el transporte de valores, escoltas personales, e incluso, seguridad de la información en la red. Cuando se trata de situaciones que puedan poner en riesgo la vida e integridad de las personas se justifica que el proceso de selección sea lo más confiable posible, ya que cualquier sacrificio a un derecho fundamental del aspirante a ser seleccionado para el trabajo de protección estaría justificado en tanto se busca garantizar derechos fundamentales de rango superior. Cabría preguntarse si llegaríamos a la misma conclusión cuando

3 "Santos denuncia una red de corrupción en la DIAN". En: Diario El Espectador, edición del 31 de marzo de 2014. "El "coco" que investiga la corrupción en la DIAN". En: Revista Semana, edición del 6 de noviembre de 2014. "Captura a 19 personas por desfalco en la DIAN”. En. El Universal, edición del 15 de noviembre de 2013. Entre muchas otras noticias sobre escándalos de corrupción en la DIAN 
el personal de seguridad que se va a seleccionar estará destinado al cuidado de bienes materiales. No obstante, la idea no es proponer juicios de constitucionalidad en situaciones tan abstractas, simplemente, en primer término, es sugerir algunas preguntas que buscan orientar una mayor profundidad en el análisis jurídico a la prueba de selección de personal mediante polígrafo, y en segundo, mostrar lo necesario que resulta evitar las generalidades provocadas al analizar los límites del uso del polígrafo para el proceso de selección de personal.

Aparte de los dos casos seńalados, no existe en el derecho colombiano ninguna otra norma expedida por el legislador en la materia, pese a los intentos de regulación al respecto, como el Proyecto de Ley 048 de 2012 que establecía en su artículo $5^{\circ}$ lo siguiente: "Con el objeto de garantizar los Derechos fundamentales, los psíquicos y emocionales de las personas, se prohíbe el uso del polígrafo para una vinculación laboral tanto en el sector público como privado" (Gaceta del Congreso, 2012). Este proyecto fue retirado del trámite legislativo sin convertirse en ley de la República, manteniendo el vacío legal respecto al uso del polígrafo en los procesos de selección.

Ante esta incertidumbre, algunos particulares han elevado consultas ante el poder ejecutivo con el ánimo de establecer si es jurídicamente admisible emplear el polígrafo en un proceso de selección. Al respecto, el Ministerio de Trabajo, mediante concepto de 8 de junio de 2011, señaló que la prueba del polígrafo no guarda relación con la valoración de las capacidades y aptitudes laborales del aspirante que el reclutador debe observar en el proceso de selección. Sostuvo, que someter a un aspirante a la prueba de polígrafo puede vulnerar principios fundamentales consagrados en el Constitución Política, como el que establece que las actuaciones de los particulares y de las autoridades públicas deberán ceñirse a los postulados de la buena fe (artículo 83 de la Constitución Política), o el que consagra que el contrato de trabajo debe ejecutarse de buena fe (artículo 55 de la misma Constitución). Tres años más tarde, el mismo Ministerio de Trabajo reiteró lo señalado en el concepto de 2011, no obstante lo cual precisó lo siguiente:

Puestas así las cosas, como se indicó al inicio, no existiendo prohibición expresa para el uso de la prueba del polígrafo sobre un trabajador, el empleador 
podría aplicarla siempre y cuando cuente con el consentimiento manifiesto del trabajador, el cual debe ser libre de cualquier vicio o coacción.<smiles>C1CCCC1</smiles>

No encontrándose su práctica prohibida por la ley, podrían practicarla siempre y cuando se cuente con la autorización escrita de la persona a examinar respetándose así sus derechos fundamentales entre los cuales, por supuesto, se encuentra la dignidad humana. (Concepto del Ministerio de Trabajo No 53266, 31 de marzo de 2014)

El Ministerio de Trabajo no es explícito en prohibir el uso del polígrafo en un proceso de selección y se limita a señalar que se debe contar con la autorización del aspirante, dejando a la presunción de autonomía de la voluntad la legitimidad de la prueba, desconociendo de esa manera que la autonomía de la voluntad es un principio fuertemente relativizado en el campo de las relaciones laborales. El giro dado por el Ministerio en tres ańos es notorio: pasa de señalar que la prueba del polígrafo no guarda relación con la valoración de las capacidades del aspirante, a admitir su práctica sin más exigencia que la manifestación de aceptación por parte del mismo aspirante. Es imposible saber la razón del cambio de criterio ante la ausencia de argumentos que lo justifiquen. Tampoco invoca el Ministerio de Trabajo algún tipo de prueba técnica que permita establecer la capacidad del polígrafo para valorar las capacidades del aspirante, por lo que no cabe otra conclusión que el cambio de criterio es cuando menos arbitrario.

Entre los pronunciamientos de los jueces laborales tampoco es posible encontrar una respuesta asertiva al problema planteado. La Sala Laboral de la Corte Suprema de Justicia, órgano judicial competente para fijar la interpretación de las leyes laborales en Colombia, no ha emitido ningún pronunciamiento que permita establecer si es admisible o no el uso del polígrafo en los procesos de selección. No obstante, existe una sentencia de la Sala Penal de la Corte Suprema de Justicia ${ }^{4}$ que puede dar pistas sobre el sentir de los jueces al respecto:

4 Esta Sala, a diferencia de la laboral, se encarga de unificar la interpretación de las leyes penales. 
(la Sala Penal de) la Corte encuentra peligros enormes frente a la libertad y a la dignidad del sujeto si se admite la utilización del polígrafo como medio de prueba, pues antes que matizar la tensión entre la finalidad del proceso como método de aproximación a la verdad y la de proteger la integridad de los derechos fundamentales comprometidos, contribuye a afianzar más el fin que los medios, debido al dramático proceso de instrumentalización a que se somete a la persona, de quien se extraen mediciones tomadas del monitoreo de las reacciones del sistema nervioso autónomo, para convertir al propio individuo en instrumento de corroboración de una verdad a la que debe llegar la administración de justicia con absoluto respeto por la dignidad humana. (Corte Suprema de Justicia, 2008)

Los argumentos de la sentencia aluden al elemento transversal en materia de interpretación de los derechos humanos: la dignidad de la persona. La Sala Penal de la Corte proscribe la instrumentalización del ser humano en la búsqueda de la "verdad", en armonía con la prevalencia de la libertad personal y otros derechos humanos. Si bien tales elementos de valoración no son ajenos al derecho laboral ${ }^{5}$, se trata de un pronunciamiento judicial cuyo acatamiento no le es exigible a quien realiza el proceso de selección, como tampoco a los jueces laborales, dado que la misma sentencia establece que los motivos por los cuales se descarta el uso del polígrafo en el proceso penal nada tienen que ver con su empleo en otras áreas, como los procesos de selección.

Sin embargo, pese a que podría sostenerse que la Sentencia de la Sala Pena de la Corte Suprema de Justicia no constituye un precedente que vincule a los jueces laborales con la tesis según la cual estaría proscrito el uso del polígrafo, el pronunciamiento citado aporta pistas importantes para encontrar una respuesta distinta a la hallada hasta ahora, una respuesta que dé prevalencia a la dignidad humana y los derechos fundamentales, aspecto que se desarrollará en el siguiente apartado.

5 Como lo muestra el contenido del artículo 57 del Código Sustantivo del Trabajo, que obliga al empleador a guardar absoluto respeto de la dignidad personal del trabajador, de sus creencias y sentimientos; así como el artículo 59 ibídem, que prohíbe a los empleadores ejecutar o autorizar cualquier acto que vulnere o restrinja los derechos de los trabajadores o que ofenda su dignidad. 


\section{Uso del polígrafo y garantía de los derechos fundamentales}

Ninguno de los tres poderes públicos constituidos institucionalmente en Colombia señala de manera expresa prohibición alguna respecto del uso del polígrafo en los procesos de selección, dejando así una ausencia normativa al respecto. Si bien en algún punto todas las fuentes consultadas hasta ahora parecen coincidir en que el uso del polígrafo puede poner en riesgo los derechos fundamentales, instrumentalizar al ser humano y vulnerar su dignidad, aún carecemos de una respuesta concluyente para la pregunta inicial.

Algunos podrían sostener que se trata de un falso dilema, dado que si aplicamos un principio general del derecho, según el cual los particulares pueden hacer todo aquello que no esté expresamente prohibido por la ley, entonces el uso del polígrafo en el proceso de selección está amparado por el derecho. Pero tal inferencia solo podría obedecer a una lectura parcial del derecho vigente. Por el contrario, una lectura constitucional del derecho, una que se tome en serio los derechos fundamentales en el trabajo, sugiere otra interpretación: el uso del polígrafo en los procesos de selección vulnera valores y principios previstos en el ordenamiento jurídico.

Según una lectura semejante, hacer lo que no esté prohibido no es una facultad absoluta, pues debemos evitar afectar con nuestras acciones los derechos humanos, aunque tales acciones puedan no estar expresamente prohibidas. La mayoría de las veces, la ley establece prohibiciones precisamente para proteger los derechos humanos, pero no siempre es así, pues el derecho constituye un lenguaje abierto, y por ende, no está exento de vacíos, anfibologías, vaguedades o cualquier otra carencia propia del lenguaje. A menudo, el derecho no prohíbe taxativamente una conducta, ni establece una sanción en caso de su ocurrencia, provocando la necesidad de lecturas más cuidadosas que las requeridas ante prohibiciones claras y expresas.

Sin embargo, aún no podemos llegar a la conclusión de que el uso del polígrafo en los procesos de selección puede constituir una vulneración a los derechos fundamentales, y por ende al orden constitucional, sin estudiar el caso de una manera más profunda y concreta. Es necesario precisar algunos aspectos sobre el uso del polígrafo en los procesos de selección con base en los estudios técnicos elaborados al respecto. 
Partamos de considerar que el proceso de selección para el empleo, aunque se haga de manera técnica y conforme a los códigos éticos y deontológicos de la psicología, es una intervención en el cuerpo del sujeto, una intrusión en su intimidad. Dentro de los proceso de intervención para la selección de personal, la psicología reconoce las pruebas técnicas como el Wartegg, el 16pf, el assessment, la visita domiciliaria, el uso del polígrafo, entre otros. De acuerdo con la investigación adelantada por Peralta (2015):

El uso del polígrafo "permite conocer la verdad de las personas", en los procesos de selección, en la medida en que "[...] mide los cambios en datos biológicos que pueden ser consecuencia de emociones" (Clemente, 2011, p. 79); cambios que a su vez son registrados en gráficas que muestran las variables fisiológicas: presión arterial, ritmo cardiaco, respuesta galvánica de la piel y la respiración (Saldaña, 2012). A través de esta herramienta, se registran las variaciones psicofisiológicas de las personas a fin de revelar inconsistencias en las respuestas o relatos de estas, al ser sometidas a una entrevista (Guerra, 2011); y con el fin de encontrar la "verdad" acerca de lo que afirman los candidatos a un puesto de trabajo y también de indagar cómo son estas personas (Sánchez, 2009). (Peralta, 2015)

El uso del polígrafo ha sido duramente cuestionado al interior de la psicología, especialmente desde los enfoques de psicología crítica, dado que el uso de esa herramienta parte de suponer la existencia de una verdad subyacente en la psiquis del entrevistado que el polígrafo puede poner al descubierto, sin tener en cuenta que tal verdad es construido con arreglo a una serie de actos interpretativos social e históricamente considerados en una cultura determinada por la aceptación de la ciencia positiva como aquella que aporta el criterio máximo de validez.

Al respecto, Peralta (2015) señala sobre el polígrafo lo siguiente:

La máquina traduce las señales del cuerpo y los traslada a un gráfico, que a su vez es traducido por el experto en polígrafos, pero esta traducción no es un paso inalterable de significados fijos o ciertos (Gibson, 2001). A las variaciones en los flujos del cuerpo y en los cambios en el gráfico se le otorga 
un significado, y lo que los expertos hacen es proponer lo que dichos cambios "significan”. Se cuestiona así la capacidad real de la máquina para detectar la mentira o el error, justamente porque lo que esto nos está diciendo es que el uso del polígrafo se sustenta en el régimen de verdad o de conocimiento que se ha establecido socialmente y de quiénes están autorizados para hablar sobre la verdad. (Peralta, 2015, p.46)

No son entonces pocos ni menores los cuestionamientos hechos por la psicología al uso del polígrafo y las consecuencias que de él se derivan. En lo que al derecho se refiere, el polígrafo se ubica en un margen muy delgado entre la libertad del empresario de seleccionar al trabajador más confiable y los derechos fundamentales de la persona que atraviesa por el proceso de selección, por lo cual es válido acudir a los criterios de interpretación que la Corte Constitucional colombiana ha hecho sobre el contenido de los derechos humanos en el trabajo. Según el principio jurídico ya señalado, los particulares podemos hacer aquello que no esté expresamente prohibido, lo que no significa que estamos facultados para hacer, literalmente, cualquier cosa.

No es fácil establecer los límites entre lo que podemos hacer y lo que no cuando de por medio se encuentran acciones que no están expresamente prohibidas por el ordenamiento legal. Existen múltiples criterios para definir tales límites, sin embargo hay uno que puede resultar pertinente dada su legitimidad y alto nivel de aceptación en una democracia: los derechos humanos. No obstante, siguiendo a Santos (2010), es preciso señalar que los derechos humanos tienden a funcionar como particulares que se hacen pasar por universales a través del uso de la fuerza. Frente a la capacidad del universalismo para aplastar las diferencias, Santos sostiene que las formulaciones sobre las cuales se ha basado la pretensión de universalidad de los derechos humanos siguen siendo el producto de la arrogancia occidental:

El concepto de derechos humanos se basa en un conjunto bien conocido de presupuestos, todos los cuales son claramente occidentales, a saber: hay una naturaleza humana universal que se puede conocer por medios racionales; la naturaleza humana es esencialmente distinta de, y superior a, la del resto de la realidad; el individuo tiene una dignidad absoluta e irreductible que debe ser defendida frente a la sociedad y al Estado; la autonomía del individuo requiere 
de una sociedad organizada de una manera no jerárquica, como una suma de individuos libres. (Santos, 2010, p. 89)

No obstante, Santos (2010) señala que los derechos humanos también pueden ser entendidos y usados como una modalidad de cosmopolitismo subalterno, lectura en la cual deberán mediar - principalmente- circunstancias de tipo cultural. Según Santos, el único hecho realmente transcultural es la relatividad de toda cultura, lo que implica siempre el carácter diverso e incompleto de cada formación cultural. La posibilidad de que las diversas culturas con sus diferentes aspiraciones a valores fundamentales igualmente disímiles puedan ser mutuamente inteligibles está determinada por la capacidad de encontrar procedimientos hermenéuticos adecuados.

La tesis de Santos (2010) presenta limitaciones, en tanto las diversas culturas están vinculadas entre sí a través de relaciones de poder (entre otras) que se manifiestan en constantes batallas -simbólicas y físicas- por imponer sus propios valores fundamentales, entrando en liza por la interpretación, contenido y alcance de los derechos humanos. No obstante, la propuesta de Santos, según la cual los derechos humanos pueden funcionar como una forma de cosmopolitismo subalterno, es una alternativa viable, especialmente si la llevamos hasta sus más fuertes implicaciones. Los procedimientos hermenéuticos como espacios de interpretación local son viables en tanto nos pongamos de acuerdo por lo menos en un elemento cuya existencia en sí misma posea un valor absoluto; esto es, a un imperativo categórico, como el segundo de los contemplados en La Metafísica de las Costumbres: "Obra de tal modo que uses la humanidad, tanto en tu persona como en la persona de cualquier otro, siempre como un fin al mismo tiempo y nunca solamente como un medio" (Kant, 2005, p.24).

Muchas de las construcciones legales -internacionales y locales- dirigidas a regular las formas de trabajo emergentes luego del proceso de industrialización se levantaron precisamente sobre el segundo imperativo categórico kantiano, lo cual no es extraño si se tiene en cuenta que han sido las relaciones de producción capitalistas lamentablemente las más exitosas en hacer del ser humano una mercancía. No es exótico entonces el argumento que defiende la prevalencia de los derechos fundamentales en el trabajo sobre las libertades del empresario, si se tiene en cuenta 
que los primeros hacen parte de un proceso mediante el cual es posible contener el poder del mercado que puede actuar incluso en detrimento de los contenidos mínimos de justicia a los cuales el derecho no puede renunciar, al menos no si pretende ser un mecanismo de orden político social, o como lo plantea Polanyi (2003), una barrera cultural en defensa de la vida. Tampoco parece necesario discutir ahora la necesidad de recuperar la capacidad del derecho para regular el trabajo (Supiot, 2010) en tanto este sigue teniendo utilidad social (Castel, 1996).

Si como lo reconocen todas las fuentes consultadas, el uso del polígrafo coloca en riesgo los derechos humanos del aspirante dentro del proceso de selección, se me ocurren por lo menos tres argumentos de principio en sustento de tal afirmación.

\section{-Argumento analógico:}

Según la ley colombiana, el demandado en un proceso de filiación renuente a practicarse la prueba de ADN decretada por un juez, asumirá como consecuencia de su reticencia la existencia de un indicio grave en su contra. Esta interpretación fue avalada por la Corte Constitucional mediante la Sentencia C-808 de 2002. Para la Corte, al encontrarse en vilo derechos de los nińos, el sistema legal considera la renuencia como un indicio grave en contra del procesado; no obstante, obligar al demandado a tomarse una muestra de sangre o de cualquier otro tejido, es un acto que vulnera su dignidad, razón por lo cual no se le puede obligar a practicarse tal prueba. Cabe resaltar que, aun estando en juego derechos de los menores -los cuales son prevalentes-, la ley no autoriza someter el cuerpo de una persona a una prueba que implique la sujeción de su cuerpo, y solo ante la prevalencia de los derechos del menor, se admite esa reticencia como un indicio grave en contra del procesado, indicio que en todo caso puede ser desvirtuado con otras pruebas.

Sinteticemos la analogía en una pregunta y trasladémosla al caso que nos ocupa: ¿existe algún valor, principio o derecho fundamental de rango superior a la dignidad, la libertad y la intimidad personal, que justifique someter al aspirante a una prueba de polígrafo, o aún más, deducir la veracidad de un hecho porque el trabajador no se sometió a dicha prueba? Una respuesta afirmativa sería tan ilógica como aceptar dos premisas en contra del ordenamiento jurídico vigente: una, la presunción de 
culpabilidad como principio, dos, la primacía de las libertades empresariales sobre los derechos humanos. Primero: la buena fe se presume, la mala fe se prueba, establece la Constitución Política. Segundo: ningún derecho del empresario -por legítimo que sea- prevalece sobre los derechos humanos, por ende, nada justifica el sometimiento a la prueba de polígrafo; tampoco se permite a los seleccionadores derivar consecuencias adversas para el trabajador en caso de no querer sentarse ante un polígrafo.

\section{- Argumento de ponderación:}

Está claro que el uso del polígrafo implica la restricción de varios derechos fundamentales para el trabajador, entre otros, a su intimidad personal, además de la amenaza que puede implicar hacia otros derechos del mismo rango. Independiente de si la restricción es autoimpuesta, cabría discutir si el ordenamiento jurídico debe ser ciego ante la posibilidad de que un ciudadano renuncie a un derecho fundamental, a sabiendas de la existencia de ciertas condiciones materiales presentes en una economía de mercado que no se encuentra bajo el completo control de una persona sometida a un proceso de selección. Una perspectiva del derecho que trascienda el formalismo jurídico y pretenda garantizar la prevalencia de los derechos fundamentales no puede ser ciega a este elemento de la sociedad contemporánea.

Será necesario preguntarse entonces si tal medida restrictiva soporta un test de proporcionalidad como el que la Corte Constitucional plantea debe hacer, no solo el operador judicial, sino todo aquel cuya actuación pueda poner en riesgo la garantía de un derecho fundamental (Colombia, 2010).

Una media restrictiva de un derecho fundamental debe someterse, en primer lugar, al juicio de idoneidad, que podría orientarse por la siguiente pregunta: ¿la restricción de los derechos fundamentales permite realizar un fin legítimo o un interés de naturaleza constitucional? En este caso: ¿la restricción del derecho a la intimidad personal de quien se somete al polígrafo en el desarrollo de un proceso de selección permite realizar un fin legítimo o un interés de naturaleza constitucional? 
Asumamos como finalidad legítima la garantía de que el empleador pueda vincular al trabajador más confiable, caso en el cual se justificaría la medida. Sin embargo, los estudios citados muestran -como también lo señaló el Ministerio de Trabajo en el 2011- que no existe una relación de confiabilidad plena entre el uso del polígrafo y la valoración de las capacidades del aspirante para considerarlo confiable, lo cual pondría en duda la idoneidad de la restricción a la intimidad personal. En esa medida, se encuentra cuestionado el primer paso del test, lo cual descartaría la necesidad de seguir adelante con los otros dos pasos. No obstante, y con un ánimo puramente analítico, verifiquemos si la medida restrictiva cumple los otros dos pasos del test.

El segundo paso se reconoce como el juicio de necesidad de la medida. A través de este, se pregunta si existen medidas menos lesivas que la restricción de los derechos fundamentales para alcanzar el objetivo buscado. Habría que preguntarse si existe alguna otra medida que permita establecer la confiabilidad del aspirante sin que sea necesario vulnerar sus derechos fundamentales. La respuesta por supuesto dependerá de cada caso concreto, donde aspectos como la naturaleza de la labor a realizar, las funciones que deberá desempeñar, el sector para el cual se requiere la fuerza de trabajo, figuran entre otras variables a tener en cuenta. No obstante, medidas como la verificación de antecedentes, o incluso la realización de otro tipo de pruebas técnicas, constituyen mecanismos que pueden aportar criterios adecuados para determinar la confiabilidad del aspirante sin tener que recurrir al polígrafo.

Si al empleador se le exige, conforme al ordenamiento jurídico vigente, una razonabilidad constitucional en sus actuaciones, el uso del polígrafo en los procesos de selección no parece soportar un test de proporcionalidad, dado que se trata de una medida cuya idoneidad y necesidad ofrecen serias dudas.

\section{- Argumento de principio}

Pasemos al último argumento. El principio pro homine establece que en caso de dos interpretaciones divergentes en las que se encuentre en riesgo la garantía de los derechos humanos, se debe preferir aquella interpretación que los salvaguarde. Esto dijo la Corte Constitucional sobre este principio: 
Según este postulado, en la interpretación de las normas aplicables a los derechos humanos se debe privilegiar la hermenéutica que resulte menos restrictiva para el ejercicio de los mismos; este principio también es denominado cláusula de favorabilidad en la interpretación de los derechos humanos, la cual ha sido consagrada en algunos instrumentos internacionales (Colombia, 2006 )

La cláusula de favorabilidad en la interpretación de los derechos humanos señala que en caso de conflicto entre distintas normas que los consagran o desarrollan, el intérprete debe preferir la más favorable a la garantía de los mismos. Es claro que en este caso se encuentra en conflicto la libertad del empleador de someter a la prueba de polígrafo a un trabajador, y del otro lado, la vigencia de los derechos a la intimidad personal, la libertad y la dignidad humana. De alguna manera, esta discusión representa el debate más amplio sobre los derechos humanos en la interpretación del liberalismo y la que en oposición se construye al interior de una democracia social.

Al respecto, Santos (2010) señala que de las dos versiones sobre los derechos humanos predominantes en la cultura occidental deberíamos adoptar la que extiende al dominio económico y social la igualdad que el liberalismo solo considera legítima en el plano político; esto es, debemos procurar una versión de los derechos humanos más social y democrática. El principio pro homine resulta acorde a lo planteado por Santos como la necesidad de escoger entre las diferentes versiones de una determinada cultura, "aquella que represente el más amplio círculo de reciprocidad, la que va más allá en el reconocimiento del otro" (Santos, 2010, p.5).

Con todo, podría señalarse que no existe ninguna vulneración, ni siquiera una amenaza, si se toma en consideración que al aspirante se le da la opción de pasar por el polígrafo, esto es, que la decisión de someterse o no a la prueba, corresponde al candidato. Al respecto, no pueden perderse de vista las condiciones del contexto. En Colombia, dado el índice de desempleo registrado, los niveles de subempleo, informalidad, flexibilidad y precariedad existentes ¿qué posibilidad de elección ante el seleccionador tiene el aspirante? No dudo que muchas personas en Colombia y muchas partes del mundo, están dispuesta a hacer, literalmente, cualquier cosa por tener un empleo decente, incluso, sentarse ante un aparato tan singular como el polígrafo. Con todo, se puede apelar a un segundo razonamiento: es deber de quienes 
estamos inmersos en la reflexión y las prácticas laborales, hacernos la pregunta acerca de qué tan autónoma es la persona al momento de intentar acceder al mundo del trabajo, pregunta que definitivamente nunca podrá arrojar la misma respuesta, en tanto cada sujeto tiene en común con todos los demás el hecho de que está en una posición distinta.

\section{Conclusiones}

Hasta el momento, en Colombia no se encuentra regulado el uso del polígrafo como herramienta para seleccionar candidatos a ocupar un puesto de trabajo, salvo los casos regulados expresamente para la DIAN y para las empresas bajo la vigilancia de la Superintendencia de Vigilancia Privada. De lo anterior podría inferirse válidamente que, al no estar expresamente prohibido, está legalmente permitido a los particulares emplear el polígrafo como herramienta en un proceso de selección laboral.

No obstante lo mencionado, es necesario señalar que por el hecho de no estar regulado el uso del polígrafo, no se puede concluir que su uso esté permitido, dado que hay derechos fundamentales cuya vigencia podría ponerse en riesgo con su uso. Es necesario entonces, apelar a principios constitucionales que permitan garantizar la vigencia de los derechos fundamentales ante lo que puede considerarse un caso de vacío normativo, lo que no equivale a decir, inexistencia de argumento jurídico.

$\mathrm{Al}$ apelar entonces a principios de rango superior, el intérprete se encuentra ante un caso de ponderación, en el cual entran en liza las libertades de contratación conferidas al empresario frente a la garantía de los derechos fundamentales de las personas que aspiran a ser seleccionadas para un puesto de trabajo. En la relación que allí se entabla, es innegable que persiste un poder en manos de quien realiza la selección y que se ejerce frente a quien aspira a ser seleccionado, persona que a su vez tiene unas necesidades socio económicas que el trabajo le permite satisfacer. El poder de quien selecciona, implica unos deberes sociales, de allí que la Corte Constitucional exija al empresario contemporáneo una razonabilidad constitucional en todas sus acciones, dado además que la solución de los conflictos laborales es un asunto de interés público. 
Debe tenerse en cuenta que la Corte Constitucional colombiana ya se ha pronunciado respecto a la controversia suscitada entre las libertades empresariales y los derechos fundamentales del trabajador. Al respecto, ese Tribunal ha señalado que la empresa cumple una función social, no solo por su impacto en el orden económico, sino por el deber que le asiste de salvaguardar los derechos fundamentales ante el poder de subordinación que le es connatural. Incluso, en la medida que los conflictos laborales son asuntos de interés público (Colombia, 2011), se justifica la intervención del juez constitucional para salvaguardar los derechos fundamentales en el trabajo.

En un caso de ponderación como el que aquí se ha expuesto -y valga señalar, cuya solución no le compete únicamente a las autoridades judiciales, sino que es el resorte de los empresarios particulares como actores socialmente responsables que deben ser-, en la ponderación adecuada entre las libertades empresariales y los derechos fundamentales, la balanza se inclina a favor de los últimos, lo que en ningún momento significa el desconocimiento de los primeros, tan solo, su sumisión a valores superiores aceptados como las garantías mínimas que una sociedad democrática y comprometida con la vigencia del estado social debe procurar a cada ciudadano.

Se concluye entonces, que no es constitucionalmente admisible el uso del polígrafo en los procesos de selección de personal; pretensión de corrección del derecho que está siendo amenazada en la práctica del caso colombiano, por el uso generalizado que se le está dando a esta técnica cuya razonabilidad constitucional es, cuando menos, seriamente cuestionada a la luz de la dignidad humana y los derechos fundamentales en el trabajo. 


\section{REFERENCIAS}

Castel, R.. (1996). Trabajo y utilidad para el mundo. En: "Trabajar por tiempos mejores. Repensar el trabajo en siglo XXI” Colección de informes OIT. 2007. En: Revista Internacional del Trabajo, vol. 115, núm. 6.

Colombia, 2002. Corte Constitucional C-808-02.

Colombia, 2006. Corte Constitucional C-187-06.

Colombia, 2010. Corte Constitucional T-247-10.

Colombia, 2011. Corte Constitucional T-434-11.

Colombia, 2008. Corte Suprema de Justicia, Sala de Casación Penal, Sentencia de $1^{\circ}$ de agosto de 2008.

Deleuze, G. y Guattari, F. (1990). Kafka. Por una literatura menor. México: Ediciones Era S.A.

Diario Oficial No 45412 de 26 de diciembre de 2003.

Diario Oficial No 46681 de 6 de julio de 2007.

Diario Oficial No 47045 del 9 de julio de 2008.

Gaceta del Congreso No 481 de 2 de agosto de 2012.

Gaceta del Congreso No 540 de 23 de agosto de 2012.

Kant, I. (2005). Metafisica de las costumbres. Barcelona: Tecnos, Anaya.

Ministerio de Trabajo. Concepto jurídico No 162736. 
Ministerio de Trabajo. Concepto jurídico No 53266.

Peralta, V.(2015). Estudios Regionales Andinos Bartolomé de las Casas. Madrid, España.: Instituto Bartolomé de las Casas

Polanyi, K. (2003). La gran transformación. Los orígenes politicos y económicos de nuestro tiempo. México: Fondo de Cultura Económica.

Santos, B. (2010). Para descolonizar Occidente: más allá del pensamiento abismal. Buenos Aires: Prometeo, Clacso, UBA.

Supiot, A. (2010). Perspectiva jurídica de la crisis económica de 2008. En: Revista Internacional del Trabajo, vol 129, num. 2. Recuperado de http://www.ilo.org/public/spanish/ revue/download/pdf/s1supiot.pdf 